\title{
Towards Nanometer-Scale Three-Dimensional Magnetic Studies with Atomic Size Electron Vortex Beams
}

\author{
Jan Rusz ${ }^{1}$, Devendra Negi ${ }^{1}$, Lewys Jones ${ }^{2,3}$ and Juan-Carlos Idrobo ${ }^{4}$ \\ 1. Department of Physics and Astronomy, Uppsala University, Uppsala, Sweden \\ 2. School of Physics, Trinity College Dublin, Dublin 2, Ireland \\ 3. Advanced Microscopy Laboratory, Centre for Research on Adaptive Nanostructures and Nanodevices, \\ Dublin 2, Ireland \\ ${ }^{4}$ Center of Nanophase Materials Sciences, Oak Ridge National Laboratory, Oak Ridge, TN 37831, USA
}

Design of nanostructures with desired magnetic properties calls for magnetic measurement techniques capable to provide quantitative magnetic information at the nano-scale, down to atomic resolution. A promising candidate for such measurement technique is electron magnetic circular dichroism (or energyloss magnetic chiral dichroism; EMCD [1]), which is an electron counterpart of the well-established xray magnetic circular dichroism technique. Recent developments allowed to detect EMCD signals using atomic size electron beams [2,3,4] and resolve quantifiable EMCD spectra from individual atomic planes [5].

We propose to extend the ever-improving spatial resolution of the EMCD technique with a sensitivity in the third dimension, i.e., depth-resolution. We argue theoretically and demonstrate computationally that when utilizing electron vortex beams with large convergence angle [6] it is possible to achieve depthresolution in EMCD measurements by moving the focal plane through the sample.

The simulations of inelastic electron scattering were done using the combined multislice / Bloch-waves method as implemented in MATS.v2 [7]. Electron vortex beams with convergence semi-angles of 30mrad and 60mrad have been propagated through a crystal of LaMnAsO at an acceleration voltage of $100 \mathrm{kV}$. Beams were centered on an atomic column containing Mn atoms.

Figure 1 shows intensity of the manganese $\mathrm{L}_{3}$ edge integrated EMCD signal for a 30nm thick LaMnAsO crystal. The total scattering cross-section has been partitioned into contributions from layers just one unit cell thick ( $c=0.904 \mathrm{~nm}$ ) in order to probe the sources of the magnetic signal at various depths. Simulations have been performed for various values of the defocus, $\Delta f$, as indicated in the legend. The position of the peak of the magnetic signal well correlates with the defocus value, see Fig.2a. The average width of the peaks obtained via fitting them with a Gaussian was obtained as $6.8 \mathrm{~nm}$ and $1.7 \mathrm{~nm}$ for convergence semi-angles of $30 \mathrm{mrad}$ and $60 \mathrm{mrad}$, respectively. This is close to the expected depthresolutions of $7.3 \mathrm{~nm}$ and $1.8 \mathrm{~nm}$ predicted by qualitative formula $1.77 \lambda / \alpha^{2}$ [8], where $\alpha$ is the convergence semi-angle and $\lambda$ is the de-Broglie wave-length of the beam electrons. Moreover, as Fig.1a,b shows, the EMCD signal contributions far from the focal plane are close to zero. Supporting this finding, Fig.2b demonstrates for LaMnAsO crystals of various thicknesses that the EMCD signal as a function of defocus steeply decreases, when the focal plane gets outside of the crystal. These findings suggest that electron vortex beams with a large convergence angle should offer a nanometer-scale magnetic depth resolution. Signal to noise ratio analysis will be presented along with possible data analysis paths $[9,10]$. 
References:

[1] P. Schattschneider et al., Nature 441, 486 (2006).

[2] J. C. Idrobo et al., Advanced Chemical and Structural Imaging 2, 5 (2016).

[3] J. Rusz et al., Nature Communications 7, 12672 (2016).

[4] T. Thersleff, J. Rusz, B. Hjörvarsson, K. Leifer, Physical Review B 94, 134430 (2016).

[5] Z. Wang et al., Nature Materials (2018), doi:10.1038/s41563-017-0010-4

[6] O. Krivanek et al., Microscopy and Microanalysis 20, 832 (2014).

[7] J. Rusz, Ultramicroscopy 177, 20 (2017).

[8] P.D. Nellist et al., Microscopy and Microanalysis 14, 82 (2008).

[9] L. Jones et al., Microscopy (2018), doi:10.1093/jmicro/dfx125

[10] Support from Swedish Research Council, Göran Gustafsson’s foundation, Carl Tryggers foundation and SNIC (NSC Center) is gratefully acknowledged. JCI acknowledges support from the Center for Nanophase Materials Sciences, which is a DOE Office of Science User Facility.
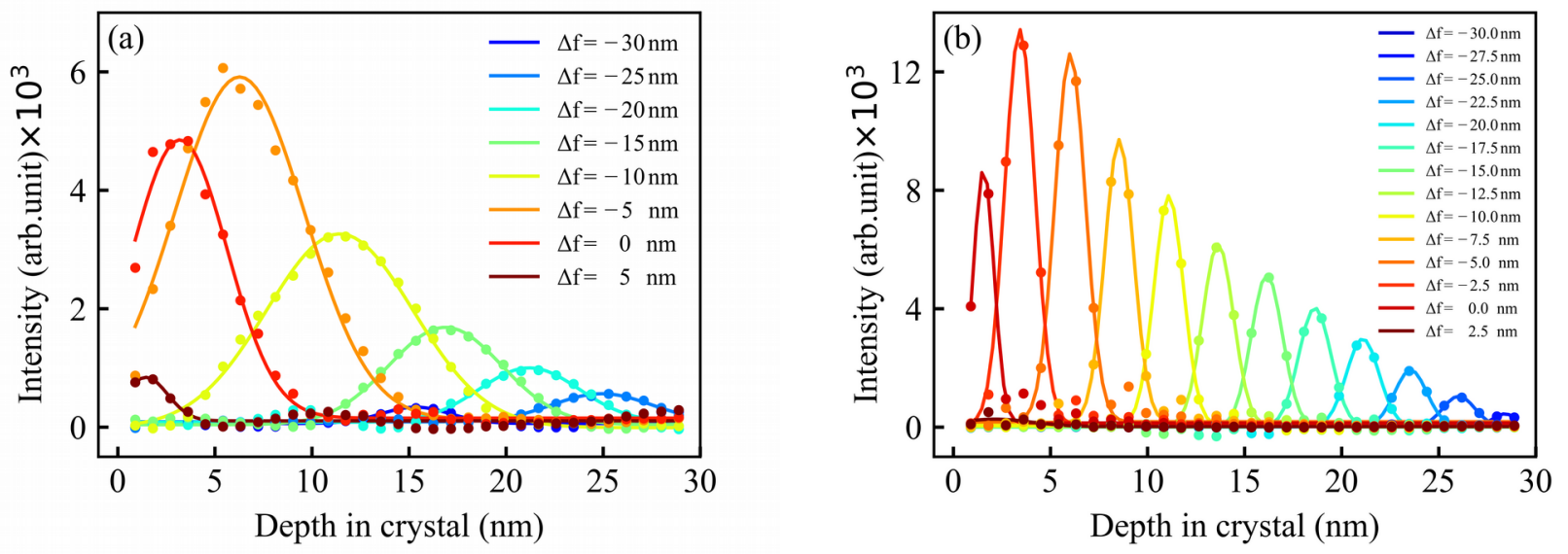

Figure 1. Depth-resolved EMCD component of the $\mathrm{Mn}-\mathrm{L}_{3}$ edge-integrated scattering cross-section for various defoci, $\Delta f$, calculated for convergence semi-angle $\alpha$ of a) $30 \mathrm{mrad}$, and b) =60mrad, respectively.
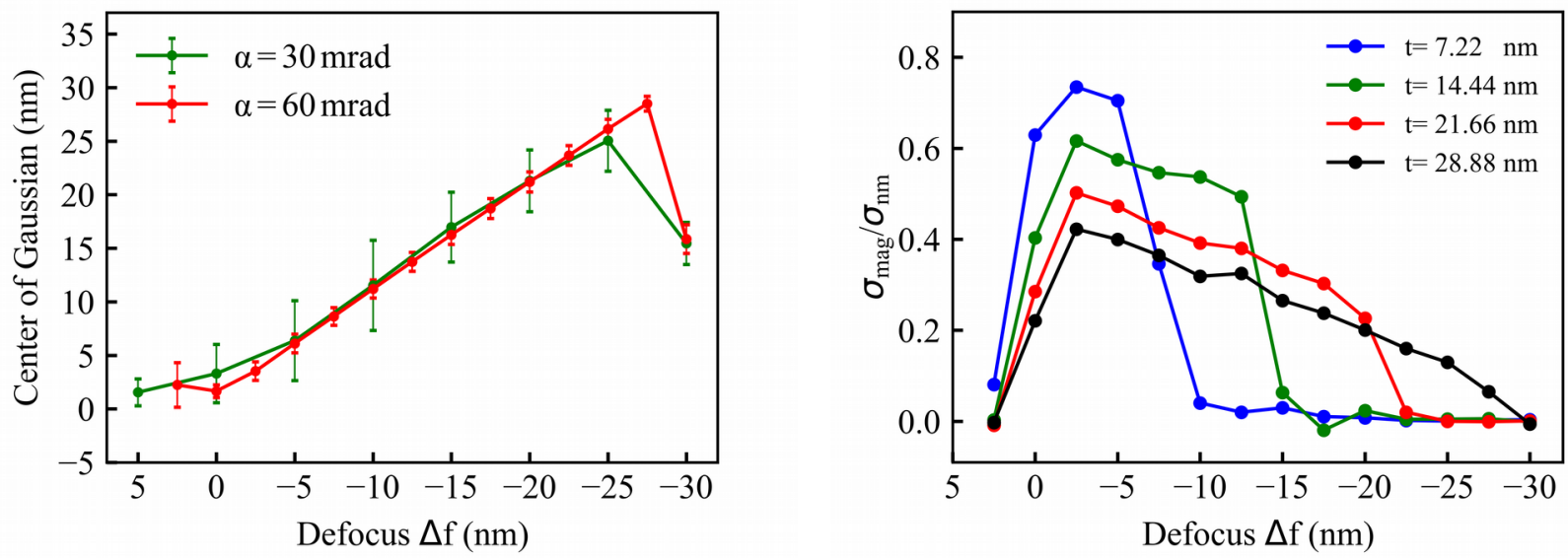

Figure 2. (a) Centers of Gaussians fitted to the profiles in Fig.1a,b and their full-width at halfmaximum (shown as error bars) as a function of defocus, $\Delta f$. (b) Total EMCD signal as a function of defocus, $\Delta f$, and sample thickness, $t$. 